\title{
Entrelacs
}

Cinéma et audiovisuel

$8 \mid 2011$

Imaginaire

\section{L'invisible... Dans le cinéma de Jean Cocteau}

\section{Rana El Gharbie}

\section{OpenEdition}

\section{Journals}

Édition électronique

URL : http://journals.openedition.org/entrelacs/226

DOI : 10.4000/entrelacs.226

ISSN : 2261-5482

\section{Éditeur}

Éditions Téraèdre

\section{Édition imprimée}

Date de publication : 1 février 2011

ISBN : 978-2-360850-03-7

ISSN : 1266-7188

\section{Référence électronique}

Rana El Gharbie, «L'invisible... Dans le cinéma de Jean Cocteau », Entrelacs [En ligne], 8 | 2011, mis en ligne le 01 août 2012, consulté le 04 mai 2019. URL : http://journals.openedition.org/entrelacs/226 ; DOI : 10.4000/entrelacs.226

Ce document a été généré automatiquement le 4 mai 2019.

Tous droits réservés 


\title{
L'invisible... Dans le cinéma de Jean Cocteau
}

\author{
Rana El Gharbie
}

« Il n'est pas impossible que le cinéma puisse un jour filmer l'invisible, le rendre visible, le ramener

à notre rythme, comme il ramène à notre rythme la gesticulation des fleurs. $»^{1}$

1 Originellement poète, Jean Cocteau fut l'un des premiers écrivains français à réaliser des films et à lancer ce défi au cinéma. Marquée par la mythologie, les rêves et le sommeil, l'œuvre de Cocteau baigne dans les gouffres infinis de l'imaginaire. Dans ses films, le poète expose son musée intérieur. De Sang d'un poète $e^{2}$ jusqu'au Testament d'Orphée $e^{3}$, Cocteau livre des autoportraits, des journaux intimes qui permettent au spectateur de rencontrer l'invisible. L'invisibilité, notion intrinsèquement liée à toutes ses créations poétiques, est le sujet principal de son œuvre cinématographique. Le cinématographe n'est pas uniquement pour Cocteau un simple véhicule ou une maîtrise de l'outil ; il s'agit d'un moyen qui permet de pénétrer dans ce no man's land qui définit l'essence de la poétique du cinéaste. Ainsi, Cocteau place les premiers piliers du cinéma d'auteur qui, non seulement se nourrit de l'âme du réalisateur, mais aussi la met en scène. Le poète filme l'invisible et assure le passage dans l'autre monde. Comment les films du poète permettent-ils au lecteur de voyager dans 1 quote invisible ? Comment le poète filme-t-il l'invisible ? Où mène-t-il le spectateur? Quel est son rôle quand il assiste au défilement des images de son imaginaire? Enfin, pourquoi Cocteau est-il hanté par la rencontre de l'invisible?

2 On pourrait s'attarder aux sujets de ses films : dans le Sang d'un poète, Cocteau filme les secrets invisibles de la création. Dans Le testament d'Orphée, il met en scène sa propre mort et son jugement dernier en tant que poète. Création et mort sont deux sujets sous-jacents de son œuvre cinématographique qui permettent au spectateur d'accéder à l'imaginaire intime du poète. En outre, le rythme de la plupart de ses films suit de près le mécanisme $\mathrm{du}$ fonctionnement du rêve. Les scènes se succèdent sans lien apparent et plongent le spectateur dans une atmosphère onirique qui l'introduit dans le mystère insaisissable de 
l'inconscient du poète. Le film est « une succession d'actes réels qui s'enchaînent avec l'absurdité magnifique du rêve $»^{4}$, souligne Cocteau. Sans oublier la symbolique du miroir, objet fréquent dans les films du poète qui permet de voyager dans son imaginaire. La traversée du miroir, scène phare dans le cinéma coctalien, assure le passage vers l'autre monde. De plus, l'image reflétée par le miroir se révèle plus vraie que la réalité. Le miroir sépare l'apparence de la vérité. Ainsi, le poète estompe les limites entre le visible et l'invisible, l'imaginaire et la réalité. Pour Jean Cocteau, «un film est une source pétrifiante de la pensée. Un film ressuscite les actes morts. Un film permet de donner l'apparence de la réalité à l'irréel. »"

\section{La théorie de l'invisible dans les écrits de Jean Cocteau}

3 La réflexion sur l'invisibilité est présente dans les journaux personnels de Jean Cocteau. Cette thématique sous-jacente est tout d'abord issue d'un problème identitaire, voire existentiel, qui se résume par une remarque du poète: «Je suis, sans doute, le poète le plus inconnu et le plus célèbre. $»^{6}$ En effet, toute sa vie, l'écrivain souffre de sa visibilité mondaine et de son invisibilité intime. En d'autres termes, Cocteau, personnage très critiqué dans la presse de son époque, est un auteur très peu lu en profondeur. Échappant à toute catégorisation, n'appartenant pas aux différents courants littéraires de son époque, refusant l'infléchissement vers un genre littéraire unique ou à un art particulier, ne s'intéressant pas à l'engagement politique qui caractérise nombre d'écrivains durant et après la Seconde Guerre mondiale, le poète reste longtemps insaisissable. La haine d'André Breton pour sa personne suscite une série de critiques dans la presse de l'époque qui le juge sévèrement. De plus, ses lettres ouvertes avec André Gide mettent en place une réputation qui dénigre l'originalité du poète. Cocteau est défini comme un prince frivole, un esprit léger qui se contente de copier sur ses amis et son œuvre est considéré e comme fantaisiste.

La constatation de son invisibilité créatrice et de sa visibilité mondaine bâtie sur des légendes et des rumeurs suscite une tristesse profonde, un sentiment de solitude excessif, une difficulté d'être insoutenable et un schisme profond entre son être et son paraître. Néanmoins, l'invisibilité, source de maux éternels, est paradoxalement un soutien pour le poète: «Et je pense que ma visibilité [...] protège mon invisibilité, l'enveloppe d'une cuirasse épaisse, étincelante, capable de recevoir impunément les coups. $»^{7}$ Cependant, Cocteau cherche à lever le masque puisqu' « être recouvert permet d'être découvert $»^{8}$. Il est important de préciser que ce dévoilement s'adresse aux futurs lecteurs qui reçoivent sur «la même longueur d'ondes " que le poète et non pas à ceux de son époque qui ne font que le décevoir et le désoler. Cocteau réussit donc à transformer cette invisibilité en une dynamique personnelle jusqu'à en faire un concept fondamental de son œuvre. L'œuvre d'art est pour Jean Cocteau substantiellement invisible. Si l'invisibilité rime avec l'incompréhension, le poète en fait l'essence de sa création puisqu'il met « au doigt de [son] œuvre l'anneau qui donne l'invisibilité $»^{10}$. Ainsi, comme il le souligne, pour le lire, il faut être un spécialiste du décryptage. Comment Cocteau nous invite-t-il à nous pencher sur ses hiéroglyphes? Cocteau choisit le cinématographe pour rendre visible son invisibilité. Il va à la rencontre de l'inconnu pour filmer l'invisible. 


\section{La création et la mort ou l'imaginaire du poète}

5 En 1930, Cocteau commence le tournage du Sang d'un poète financé par les Noailles. Ce premier film s'intéresse à la création en mettant en scène un peintre qui n'est autre que le double du poète. La voix off de Cocteau, l'insertion dans le film de ses citations et les étoiles - emblèmes de sa signature - rythment le film et rappellent la participation intime du réalisateur à son œuvre. En effet, dès les premières minutes du film, Cocteau précise qu'il s'agit de pénétrer dans un autre monde. La première image expose un personnage masqué, debout devant des caméras, qui lève le bras et nous indique la direction à suivre. S'ensuit un plan sur une porte fermée avec une clé et une poignée qui bouge. Une inscription rappelle que "Tout poème est un blason. Il faut le déchiffrer. » La note suivante souligne qu te il s'agit d'un «documentaire réaliste d'événements irréels ». Ainsi, le réalisateur introduit le spectateur dans un monde caché et secret, un univers codé qu'il faut analyser. La thématique de l'invisibilité est donc surdéterminée dès le début de ce film.

6 La mise en abyme de la création d'un film et la réflexion sur le processus de création en général caractérisent cette première œuvre cinématographique. L'invisible est donc le monde ténébreux de l'artiste au travail. Cocteau choisit de nous dévoiler cet univers par une «bande d'allégories ». Ainsi, l'hôtel des folies dramatiques où est introduit le peintre pourrait représenter l'art cinématographique que Cocteau découvre et expérimente. De même, la scène qui montre le poète en train de tricher symbolise l'origine de ses œuvres. La carte volée du manteau de l'élève tué par Dargelos - personnage fétiche de l'enfance de Cocteau qui revient dans plusieurs œuvres, notamment dans Les Enfants terribles- rappelle que «l'artiste est ce tricheur qui dépouille sa propre enfance $»^{11}$ et en fait des films. Pareillement, le premier épisode, "La main blessée ou les cicatrices du poète», qui montre à plusieurs reprises la main du poète avec une bouche qui parle en gros plan, définit la conception du cinématographe pour Cocteau: «[...] qu'est-ce qu'un poète de cinéma pour Cocteau? C'est exactement quelqu'un qui parle avec ses mains. Pour le montrer sur l'écran, sans le dire [...], quoi de plus efficace comme image, de plus net, de plus parlant qu'une main qui parle en gros plan? $»^{12}$ Ainsi, l'intérêt que Cocteau porte pour le cinématographe s'explique avant tout par sa fascination pour cet art artisanal, comme il le souligne dans ses entretiens avec André Faigneau ${ }^{13}$. Le sang d'un poète retrace l'expérience même de ce film. Par ailleurs, Cocteau y expose une de ses théories sur la création. La phénixologie, concept emprunté à Salvador Dali, est l'art de renaître de ses cendres. La mort du poète, mise en scène à deux reprises dans le film, n'est jamais une fin en soi et mène toujours au réveil. Le premier suicide du poète est dicté par la voix mystérieuse d'un bras qui lui tend un pistolet et lui dicte son mode d'emploi, la deuxième suit sa perte au jeu de cartes. La mort du poète est nécessaire à la survie de l'œuvre et survient irrévocablement à sa fin. Elle est un sacrifice indispensable pour le bon fonctionnement du processus créateur.

7 Dans cette première œuvre cinématographique, Cocteau nous invite à rejoindre ses ténèbres intimes afin de nous livrer une réflexion sur le cinéma et sur la création. L'invisibilité de l'inspiration, ou de l'« expiration » comme l'appelle Cocteau, est donc visible pour le spectateur qui, à l'image de la femme-statue dans ce film, ferme paradoxalement les yeux pour mieux voir et suit la descente du poète. 
8 Dans son dernier film, Le Testament d'Orphée, Cocteau filme sa propre mort et son jugement dernier. Cocteau commence le tournage en 1959, financé entre autres par Francine Weisweiller et François Truffaut. Ce film s'ouvre, comme le premier, sur une remarque du poète, cette fois en voix off, qui rappelle l'attachement du cinématographe à la thématique de l'invisibilité :

"Le privilège du cinématographe, c'est qu'il permet à un grand nombre de personnes de rêver ensemble le même rêve et de montrer en outre avec la rigueur du réalisme les fantasmes de l'irréalité. Bref, c'est un admirable véhicule de poésie. Mon film n'est pas autre chose qu'une séance de striptease consistant à ôter peu à peu mon corps et montrer mon âme toute nue. Car il existe un considérable public de l'ombre affamé de ce plus vrai que le vrai qui sera un jour le signe de notre époque. Voilà le lègue d'un poète aux jeunesses successives qui l'ont toujours soutenu. ${ }^{14}$

Le film tout entier est placé sous l'égide d'un voyage qui transgresse les limites spatiotemporelles. Le temps n'est plus chronologique, il n'est plus linéaire. Dans les premières scènes du film, se succèdent les images d'un professeur à l'adolescence, enfant, durant sa vieillesse puis à l'âge mûr. Les scènes, séparées par la fumée qui monte sur un fond noir, ne suivent plus un ordre diachronique et brouillent toute logique temporelle. Plus encore, à la question du professeur qui demande "Quelle heure est-il ?", l'homme de la commission d'enquête répond tout simplement : " Aucune. » L'abolition de la temporalité s'accompagne d'une réflexion sur l'espace. Le poète se trouve dans «la zone, où les vivants ne son $t$ pas vivants et où les morts ne sont pas morts ». Les frontières spatiotemporelles du voyage du poète vers son tribunal final s'estompent. Ainsi, le spectateur accompagne le poète dans ses limbes.

10 Le point de départ du poète est sans aucun doute le rêve, comme le souligne l'ouverture du film citée ci-dessus. Un rêve qui paradoxalement n'est " plus un film » mais « la vie » comme le rappelle Cégeste ${ }^{15}$ à Cocteau. Le poète inverse donc toutes les données afin d'emporter le spectateur dans un monde régi par de nouvelles lois, où l'espace et le temps importent peu, où les images défilent à l'envers, où les personnages apparaissent puis disparaissent, où les morts ressuscitent pour poursuivre les diverses épreuves de leur voyage. Cocteau questionne le rapport entre le réel et l'imaginaire. Il prône cette faculté fantasmagorique et choisit de filmer ce monde intermédiaire qui sépare le réel de l'irréel.

11 Le Testament d'Orphée représente les images de la cosmogonie du poète, son musée intérieur, son imagination en œuvre, sa mythologie personnelle. Accusé lors de son procès de « vouloir pénétrer sans cesse dans un monde qui n'est le [sien] », le poète plaide coupable et s'explique :

"[...] j'avoue vouloir souvent sauter le quatrième mur mystérieux sur lequel les hommes écrivent leurs amours et leurs rêves [...] sans doute par fatigue du monde que j'habite et par horreur des habitudes, aussi par cette désobéissance que l'audace oppose au rêve et par cet esprit de création qui est la plus haute forme de l'esprit de contradiction propre aux humains. $»^{16}$

Coupable, le poète l'est, puisqu'il baigne dans le monde de la poésie. Le dernier film de Cocteau, son requiem, est la représentation de la poésie même : «'Avec le film, on tue la mort, on tue la littérature, on fait vivre la poésie d'une vie directe.' Le cinéma fait donc plus que 'construire un véhicule à la poésie'. Il la 'réalise' [...]. ${ }^{17}$ L'image de l'hibiscus qui traverse tout le film et qui est sa "vedette », est emblématique de la posture créatrice de Cocteau, laquelle consiste, non pas à raconter, mais à montrer la poésie en œuvre. Le gros plan sur les mains de Cocteau qui ressuscite la fleur détruite et écrasée est l'une des plus belles scènes du cinéma $d u x x^{~}$ è siècle. Cette fleur, que le poète n'arrive pas à dessiner 
puisqu'il est condamné à tracer éternellement son autoportrait, revit entre les mains de Cocteau qui ne fait qu'obéir aux ordres de Cégeste. Le poète, serviteur d'une force inconnue qui le hante, crée ou plutôt redonne le souffle de la vie à une image morte enfouie dans ses souvenirs et ses fantasmes, tout cela pour l'offrir à la juge qui le condamne d'innocence. Cette femme au regard sévère et à la voix grave représente les contemporains de Cocteau qui ne cessent de le critiquer, comme nous l'avons vu. Elle pourrait aussi rappeler que ce jugement qui le hante dans sa vie visible, c'est-à-dire mondaine, pénètre dans son invisibilité et que Cocteau finit lui-même par s'accuser d'être un poète. Cocteau reprend la fleur sous les ordres de la juge. Par la suite, il la laisse tomber de ses mains quand il est assassiné par l'épée de la déesse. La caméra se pose tout de suite sur l'image de la fleur qui gît à côté de la flaque du sang de Cocteau. Puis, miraculeusement, la fleur et le sang se colorient en rouge. Cette unique apparition d'une couleur dans le film tisse un lien intime entre la fleur et le sang, en d'autres termes entre la poésie et l'« âme toute nue » du poète. Ainsi, Cocteau sacrifie son sang au règne de la poésie. L'investissement du poète est donc incontestable et son engagement dans son œuvre est sans équivoque. Enfin, la fleur est laissée dans la poussière sous les pieds des " policiers de la route ». Ces derniers demandent les papiers de Cocteau ressuscité qui est alors emporté par Cégeste. Le poète disparaît et la fleur gît sur le sol, abandonnée par tous, inaperçue. Cocteau nous rappelle que sa poésie, qui lui survivra, qui restera de ce monde alors que lui s'en sera allé pour toujours, est injustement négligée. Ne veut-il pas que le spectateur ramasse cet hibiscus? Le point d'arrivée du poète est sa propre mort, sa résurrection et enfin sa disparition puisque «la terre, après tout, n'est pas [sa] patrie ». À la fin du film, le poète n'est ni mort ni vivant, il n'est ni ici ni ailleurs. Il part avec Cégeste rejoindre peut-être les éternels anges qui hantent sa poésie.

Le poète invite le spectateur à l'accompagner dans un voyage vertigineux dans l'invisible et à explorer sa poésie dont "l'exhibitionnisme s'exerce chez les aveugles »" Le rythme de cette traversée qui oscille entre les frontières incertaines du réel et de l'imaginaire, qui «brouill[e] l'ordre des facteurs auquel la raison nous condamne ${ }^{19}$, est en désordre. Le rythme des films de Cocteau suit celui d'un rêve.

\section{Le rythme des films ou le mécanisme du rêve}

Cocteau s'intéresse au monde des rêves. Cependant, il relate très rarement les récits de ses rêves dans ses journaux personnels. Ce qui le passionne dans cette dynamique imaginaire, c'est le rythme même du rêve, l'enchaînement des images qui défilent en dehors de toute logique apparente. Le poète utilise le mécanisme du rêve et le transpose dans ses films, notamment dans le Sang d'un poète et le Testament d'Orphée. Contrairement à ses autres œuvres, ces deux films ne relatent pas une histoire et ne doivent pas obéir aux règles d'agencement des scènes, ils sont «débarrassé[s] de tout souci référentiel anecdotique $»^{20}$. Le poète rompt ainsi la chaîne de cause à effet et privilégie le hasard par rapport aux faits tangibles. Le voyage filmé est donc l'odyssée du dormeur. Dans la Difficulté d'être, le poète définit les caractéristiques du rêve qui rappellent inévitablement celles de ses films :

"Le passé, l'avenir n'existent plus, les morts ressuscitent, les lieux se construisent sans architecte, sans voyages, sans cette lourdeur lente qui nous oblige à vivre minute par minute ce que la pliure entrouverte nous montre d'un seul coup. En outre, la légèreté profonde et atmosphérique du rêve favorise des rencontres, des surprises, des connaissances, un naturel, que notre monde plié (je veux dire projeté sur la surface d'une pliure) ne peut mettre que sur 
le compte du surnaturel. Je dis naturel, car une des caractéristiques du rêve est que rien ne nous étonne. [...] Le spectacle du sommeil m'a toujours plus effrayé que le rêve. $»^{21}$

$$
\text { à l' }
$$
chacune des portes. Dans la première, un Mexicain se fait tirer dessus et tombe au ralenti. La scène s'inverse et l'homme se retrouve debout. Par la suite, le poète voit la même scène pour la deuxième fois. La deuxième porte s'ouvre sur un jeu d'ombres qui montre la préparation d'une pipe d'opium et une fumée qui s'en dégage. La troisième chambre est habitée par une jeune fille et sa maîtresse qui lui apprend la « leçon du vol ». La fille lui fait une grimace du plafond. Dans la dernière chambre se donnent les rendez-vous d'hermaphrodites. Sur un canapé, les membres d'un personnage étrange surgissent un par un jusqu'à l'apparition sur son sexe d'une pancart e qui indique " Danger de mort ». Comment expliquer le lien entre ces quatre scènes? Comment justifier le passage de l'une à l'autre ? Quel est rapport entre ces personnages énigmatiques espionnés par le poète ? Afin de comprendre cet étrange enchaînement d'images sans rapport apparent, nous devons revenir à l'univers intime du créateur. La phénixologie, l'opium, la désobéissance et l'ambiguïté de la sexualité sont des thèmes primordiaux dans l'univers coctalien. Ces thématiques, représentées dans les chambres de cet hôtel, soulignent que le seul ordre qui pourrait régner est celui de l'invisibilité et que la ligne qui maintient l'unité de ces images fragmentaires est tracée par le sang même du poète. Comme le montre très bien Serge Linarès dan s son ouvrage La ligne d'un style (1999, Paris, CDU SÉDÈs), Cocteau se distingue des surréalistes par le fait que le rêve est le fonctionnement secret de l'être, en d'autres termes son état normal. La succession de ces images se déroule en suivant le mouvement du rêve. Par ailleurs, la même scène peut se rapporter à deux univers distincts. Par exemple, la première chambre pourrait rappeler le suicide de son père sans cesse répété, comme elle pourrait faire référence au concept de phénixologie. De même, le fumeur dans la deuxième chambre représente la période d'intoxication du poète d'une part et l'atmosphère même de ce film qui joue le rôle d'une désintoxication et qui lui permet d'exorciser ses démons. En effet, si les images opiacées ne font plus partie du quotidien du poète, Cocteau décide de baigner son film dans une fumée oppressante et libératrice. Tourné après sa désintoxication, Le Sang du poète est tout entier sous le signe de cette drogue dont la fumée saccade les différentes scènes. La fumée apparaît au début du film en alternance avec l'image d'une porte qu'on essaye d'ouvrir. Cocteau rappelle ainsi que les interprétations sont infinies, à l'image du rêve d'un dormeur ou d'un opiomane. Issues des souvenirs enfouis du poète ou de ses concepts de créateur, ces scènes sont un condensé de l'imaginaire coctalien. Ainsi, l'ordre visible est remis en question afin de célébrer une logique interne, celle de l'inconscient. Cocteau mêle alors sa vie personnelle et sa vie d'artiste : « [Son] sang est devenu de l'encre. $»^{24}$ La transposition du mécanisme du rêve dans ses films édifie une "architecture cachée » et donne «à l'œuvre, boiteuse en apparence, une harmonie $»^{25}$ secrète. Cocteau ne se contente pas de filmer les images de son imaginaire, il donne au rythme de ses films l'essence même de cet imaginaire. L'ordre invisible est donc celui de sa ligne interne qu'il définit comme « la permanence de [sa] personnalité », comme "le style de l'âme ${ }^{26}$. Du Sang du poète au Testament d'Orphée, Cocteau utilise le miroir et transforme cet objet du quotidien en un 
objet poétique par excellence. Quel est le rôle du miroir dans les films de Cocteau ? L'image reflétée dans le miroir nous permet de nous interroger sur la dialectique de la vérité et de l'illusion, du visible et de l'invisible, du réel et de l'imaginaire.

\section{Le miroir ou le passage vers la zone}

17 Premièrement, le miroir révèle la vérité. Il reflète la réalité qui est au-delà des apparences. Il ne se contente pas de réfléchir l'image mais réfléchit à son tour, comme le souligne à plusieurs reprises le poète. Ce jeu d'homonymes rappelle le pouvoir magique des miroirs qui privilégient la réflexion d'une image invisible, laquelle - contrairement à l'image qui est censée se refléter - est réelle. Ainsi, le miroir montre le fond de l'âme ${ }^{27}$. Par exemple, les sœurs de Belle en se regardant dans le miroir envoyé par la Bête voient une vieille dame et un singe. ${ }^{28}$ Par ailleurs, le miroir peut refléter une réalité invisible que désire voir celui qui s'y contemple. Belle voit dans le miroir son père puis la bête en souffrance. L'image reflétée par le miroir dépasse donc le masque qu'est le corps afin de montrer l'essence de l'être. Cocteau remet alors en question la dualité entre l'être et le paraître. Le miroir refuse de renvoyer l'image qui est jugée trop visible, trop irréelle et reflète donc la réalité invisible. Cocteau trompe l'apparence pour montrer sa profondeur. Deuxièmement, le miroir est un moyen de transport. Étroitement lié à la mort et au temps qui passe, le miroir permet de voyager dans les ténèbres de l'imagination ou d'accéder à la « zone ». Dans Le Sang d'un poète, le poète est invité par la statue à pénétrer dans le miroir. Il flotte dans le noir et nage au ralenti avant d'arriver à l'hôtel des FoliesDramatiques. Ce voyage permet au poète de se promener dans ses souvenirs, comme nous l'avons vu plus haut, afin de déchiffrer ses propres secrets. Dans Orphée, Heurtebise livre le secret des miroirs à Orphée :

"Je vous livre le secret des secrets, les miroirs sont les portes par lesquelles la mort vient et va. [...] Du reste, regardez-vous toute votre vie dans un miroir et vous verrez la mort travailler comme les abeilles dans une ruche en verre. $»^{29}$

18 La mort utilise cet instrument pour pénétrer dans le monde des humains. De même, Orphée l'utilise pour aller à la recherche d'Eurydice. La « zone » est un espace dépourvu de toute perspective, où la loi de la pesanteur n'agit plus, où le temps est immobilisé. Elle est « faite des souvenirs des hommes et des ruines de leurs habitudes ». La « zone », dans Orphée et dans le Testament d'Orphée, est là où le poète est jugé. Ainsi, Cocteau rappelle que l'ambiguïté entre le visible et l'invisible, entre le paraître et l'être, est intrinsèquement liée au tribunal qui condamne les poètes d'innocence et qui leur demande de définir la poésie. Hanté par les critiques sur sa personne tout au long de sa vie, Cocteau s'explique et assure sa défense lors de procès imaginaires auxquels il accède grâce à la traversée d'un miroir. En d'autres termes, ce passage souligne que le paraitre du poète importe peu et qu'il faut aller à la recherche de son invisibilité afin de percevoir sa ligne intime.

19 Le miroir se brise souvent dans Orphée et révèle ainsi son pouvoir de métamorphose et d'éclatement. L'image n'est plus unique, ni authentique, elle est multipliée à l'infini et déformée. Le miroir brisé montre la réalité de l'être fragmenté et pluriel. Cocteau met en valeur donc ses images innombrables, brouille les limites entre l'image et son reflet et invite le lecteur à le rejoindre dans une "réalité kaléidoscopique $»^{30}$ :

«Loin de n'avoir qu'une fonction contemplative, c'est par le miroir en effet que s'effectue la descente dans les profondeurs et que le reflet, loin de figer l'image du double, l'ouvre, la creuse, la lance vers le travail de métamorphose... Objet 
essentiellement transitionnel, le miroir révèle l'apparence de l'identité, la vérité du

double obscur, de l'alter ego ${ }^{31}$. »

\section{Conclusion}

20 notamment de son cycle orphique ${ }^{32}$, baignent entièrement dans l'imaginaire du poète cinéaste. Ils rappellent sa difficulté d'être et analysent les secrets de son mécanisme créateur. Accusé d'être excessivement visible, le poète rectifie son image trop apparente, trop irréelle. Il demande au spectateur de rendre visible son invisibilité, de mettre en avant son être et de condamner son paraitre.

La motivation principale de Cocteau est de se justifier, de s'expliquer, de s'exposer, de se rendre visible, c'est-à-dire de montrer son invisibilité. Il veut avant tout être cru. Cependant, son entreprise dépasse son but premier et le poète met en place une réflexion sur la dualité entre l'image et l'imagination. Où s'achève la rélité ? Où commence le reflet ? Cocteau estompe les limites entre le réel et l'imaginaire, crée le réalisme irréel, le "plus vrai que le vrai " $^{33}$ et rappelle que " le réalisme est la seule mesure dans laquelle il nous soit permis de percevoir l'irréalité $»^{34}$. L'image dans les films de Cocteau n'est pas située entre le réel et l'imagination, elle est une condensation des deux, une sur-réalité propre au poète. L'image est poésie, en attente d'être découverte et déchiffrée. Le spectateur, en regardant les images du film projeté, plonge dans cet écran, à l'image d'Orphée qui glisse dans le miroir, et accède à cette zone où il risque de rencontrer le poète et de croiser au passage sa propre mort.

\section{NOTES}

1. Jean Cocteau, Opium Journal de désintoxication, Paris, Stock, [1930], 1999, page 151.

2. Le Sang d'un poète, 1930/1932, France, $49 \mathrm{mn}$, réalisation : Jean Cocteau, production : Vicomte de Noailles.

3. Le Testament d'Orphée, 1959/1960, France, 77mn, réalisation : Jean Cocteau, production : Jean Thuillier, Les Editions cinématographiques / Les films du Carrosse.

4. Jean Cocteau, Entretiens sur le cinématographe, Monaco, Éditions du Rocher, 2003, page 47.

5. Testament d'Orphée, op.cit.

6. Jean Cocteau, Journal d'un inconnu, Grasset et Fasquelle, [1963], 2003, page 24.

7. Journal d'un inconnu, op. cit., page 24.

8. Jean Cocteau, Le Passé défini, tome I, 1951-1952, Gallimard, 1983, page 164.

9. Ibid., page 206 .

10. Jean Cocteau, Le Passé défini, tome V, 1956-1957, Gallimard, 2000, page 513.

11. Philippe Azoury et Jean-Marc Lalanne, Cocteau et le cinéma, Cahiers du cinéma, 2003, page 28.

12. Maurice Mourier, "Cocteau poète dans le cinéma ou poète du cinéma ", Le cinéma de Jean Cocteau, suivi de Hommage à Jean Marais, Actes du colloque de Montpellier 13 et 14 mai 1993, Textes réunis par Christophe Rolot avec des textes retrouvés et des inédits de Jean Cocteau 
réunis par Pierre Caizergues, Montpellier, Centre d'Etudes Littéraires Françaises du xx è siècle, Université Paul-Valéry, 1994, pages 117,8.

13. Op. cit., page 44 .

14. Le Testament d'Orphée, op.cit., nous soulignons.

15. Cégeste est un personnage du Testament d'Orphée qui guide Cocteau dans ce voyage.

16. Le Testament d'Orphée, op. cit.

17. Michel Décaudin, «Une œuvre clé : Le Sang d'un poète », Le cinéma de Jean Cocteau, op. cit., page 143.

18. Jean Cocteau, Journal d'un inconnu, op. cit., page 17.

19. Jean Cocteau, La Difficulté d'être, Editions du Rocher, [1983], 1989, page 68.

20. Laurence Schifano, Orphée de Cocteau, Atlande, 2002, page 31.

21. Jean Cocteau, La Difficulté d'être, op. cit., page 67.

22. Ibid., page 68 .

23. «Dans le Sang d'un poète, le poète progresse difficilement d'une porte à l'autre du couloir des Folies-Dramatiques, comme si les coordonnées de l'espace avaient été renversées - de fait, il évolue sur un décor posé au sol, rabattu sur le plan. ", Catalogue de l'exposition "Jean Cocteau, sur le film du siècle ", Éditions du Centre Pompidou, 2003, page 32.

24. Jean Cocteau, Euvres poétiques complètes, Gallimard, 1999, page 540.

25. Ibid., préface du Cap de Bonne Espérance, page 6.

26. Jean Cocteau, La Difficulté d'être, op. cit., page 158.

27. Stanislas lit à la reine un passage de Hamlet: "Hamlet, Asseyez-vous, vous ne bougerez pas, vous ne vous en irez pas avant que je vous ai présenté un miroir où vous puissiez voir le fond de votre âme. ", Voir L'aigle à deux têtes, 1947, France, $88 \mathrm{mn}$, réalisation : Jean Cocteau, production : Ariane Films, Sirius.

28. La Belle et la Bête, 1945/1946, France, 100 mn, réalisation : Jean Cocteau, production : André Paulvé.

29. Orphée, 1949/1950, France, $112 \mathrm{mn}$, réalisation : Jean Cocteau, production : André Paulvé et Films du Palais-Royal.

30. Catalogue de l'exposition "Jean Cocteau, sur le film du siècle ", op. cit., page 32.

31. Laurence Schifano, Orphée de Cocteau, op. cit., page 67.

32. Le Sang d'un poète, Orphée et Le Testament d'Orphée.

33. Voix off, ouverture du Testament d'Orphée

34. Jean Cocteau, Le Passé défini, tome III, 1954, Gallimard, 1989, page 56.

\section{RÉSUMÉS}

La thématique de l'invisibilité hante l'œuvre cinématographique de Jean Cocteau. Le poète développe une théorie de l'invisible dans ses journaux personnels. En effet, il transforme son invisibilité créatrice, source de maux éternels, en une dynamique personnelle et en un concept fondamental de sa création. Cocteau choisit le cinéma pour rendre visible l'invisible. Ses films convergent toujours vers deux thématiques essentielles: la création et la mort. Le cinéaste permet donc aux spectateurs d'accéder à son imaginaire intime et d'explorer sa poésie. Les films de Cocteau, notamment son cycle orphique, suivent le mécanisme du fonctionnement du rêve. Une fois l'invisibilité rendue visible, la difficulté d'être exprimée et les secrets du mécanism e 
créateur dévoilés, Cocteau rectifie sa figure mondaine, se dénude et signe une image cinématographique sur-réelle.

\section{AUTEUR}

RANA EL GHARBIE

Doctorante, Université Paris IV, Sorbonne 